Trauma Berufskrankh 2007 - 9[Suppl 3]:

S323-S328

DOI 10.1007/s10039-007-1231-6

Online publiziert: 31. Mai 2007

(c) Springer Medizin Verlag 2007

\author{
N. Kohnen ${ }^{1,2}$ \\ ${ }^{1}$ Institut für Geschichte der Medizin, Universität Düsseldorf \\ ${ }^{2}$ Köln
}

\section{Schmerzliche und nichtschmerzliche Patienten}

\section{Transkulturelle Aspekte des Schmerzerlebens}

\section{Schmerzempfinden, Schmerzschwelle und Schmerztoleranz in anderen Kulturen}

Schmerzen werden in verschiedenen Kulturen unterschiedlich wahrgenommen und gedeutet, die Empfindungs-/Reizschwelle dagegen ist bei allen Völkern gleich.

Sternbach u. Tursky [11] führten Empfindungsschwellenmessungen bei Frauen verschiedener ethnischer Gruppen (Italienerinnen, Jüdinnen, Irländerinnen und Frauen alteingesessener amerikanischer Familien) durch, ohne signifikante Unterschiede zwischen den Gruppen festzustellen. Melzack [9] deutete dies als Hinweis, dass der sensorische Leitungsapparat bei allen Menschen ähnlich funktioniere.

Die Schmerztoleranz dagegen ist in verschiedenen Kulturen unterschiedlich. Nach Sternbach et al. [12] zeigen Italienerinnen die niedrigsten Schmerztoleranzwerte. Italienische und jüdische Frauen demonstrierten deutliche Schmerzreaktionen, während amerikanische und irische Frauen durch eine wesentlich höhere Schmerztoleranz auffielen.

Auch die Schmerzschwelle ist kulturell unterschiedlich. Sie ist der Punkt, an dem eine Person einen Stimulusreiz als schmerzhaft empfindet. Clark u. Clark [2] benutzten elektrische Reize zur Schmerzschwellenmessung und stellten fest, dass bei nepalesischen Trägern (Sherpas) und bei westlichen Touristen die Empfindungsschwelle gleich hoch war, dass die Nepalesen allerdings erst bei einem hö- heren Stimulus den Reiz als schmerzhaft empfanden.

Nach Hardy et al. [3] werden Hitzereize von Ethnien, die aus dem Mittelmeerraum stammen, schon als schmerzhaft wahrgenommen, die Nordeuropäer noch als warm bezeichneten.

\section{Emotionale Faktoren des Schmerzerlebens: die Schmerzlichkeit}

In der deutschen Sprache kann zwischen dem körperlichen (schmerzhaften) und dem seelisch emotionalen (schmerzlichen) Schmerz unterschieden werden. So sagt man, dass Erblinden und Blindheit „schmerzlich“ sei, weil der Begriff „schmerzlich“ im Unterschied zu "schmerzhaft" die emotionalen Faktoren des Schmerzes benennt. Untersuchungen zum Schmerzerleben bei den Cabuntogueños, einer Fischergruppe im Süden der Philippinen, ergab, dass diese einen erweiterten Schmerzbegriff besitzen, weil sie die emotionalen Schmerzfaktoren stärker betonen als die körperlichen [5]. Die untersuchte Fischergruppe hält z. B. Erblinden, Blindheit, Taubheit und Geistesschwäche für sehr schmerzhaft.

Unsere Untersuchungen zum Schmerzbegriff in Deutschland ergaben, dass auch bei Deutschen die emotionalen Faktoren des Schmerzes einen größeren Raum einnehmen, als dies für Ärzte bisher offenbar ist. Dabei wurde nach der Schmerzhaftigkeit (nicht Schmerzlichkeit) gefragt. Die Frage lautete: „Ist Krankheit X nicht schmerzhaft, schmerzhaft, sehr schmerzhaft oder sehr sehr schmerzhaft?"

Deutsche Patienten $(n=393)$ halten Erblinden in $66 \%$ und Blindheit in 55\% für schmerzhaft (Kohnen [7], S 52-54). Nun wäre es unangebracht, der Mehrheit der Bevölkerung ein erweitertes Schmerzerleben zuzusprechen, denn wer sollte dann die Grundlage für ein normales Schmerzbefinden gründen, vielmehr ist es so, dass die naturwissenschaftlich ausgebildeten Mediziner einen rein auf den somatischen Aspekt reduzierten Schmerzbegriff haben. Ein Grund hierfür ist, dass der Schmerzbegriff im Sprachgebrauch der Ärzte operationalisiert wurde und der Schmerz im allgemeinärztlichen Gespräch in einer bestimmten Funktion und zu einem bestimmten Zweck eingesetzt wird. Er ist für Ärzte dasjenige Zeichen, durch das sie in ihrem organpathologischen Krankheitsverständnis den Ort der Gesundheitsstörung lokalisieren können. Die emotionalen Aspekte des Schmerzes werden im Arzt-Patienten-Gespräch oft ausgeklammert und sind hinderlich, wenn es darum geht, den Sitz der Krankheit anhand des Schmerzes herauszufinden.

\section{Schmerzliche Patienten}

Schmerzliche Menschen sind diejenigen, die bei der Schmerzeinschätzung von Krankheiten die emotionalen Schmerzaspekte stärker bewerten als nichtschmerzliche Menschen. Schmerzlich werden die Menschen genannt, die Erblinden, Blindheit, Taubheit und Geistesschwäche als schmerzhaft einschätzen. Der Anteil der 


\section{Körperintegrität und Schmerzwahrnehmung}

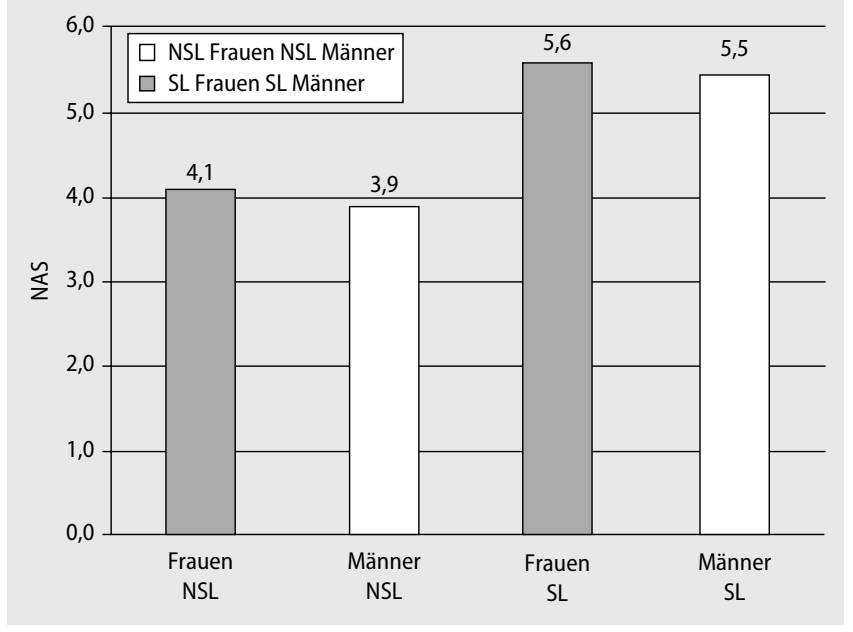

und Umwertung des Schmerzes („reframing"), Entspannungstechniken, Wärmeapplikationen, entlastende Riten, die die individuelle und soziale Dimension des Schmerzes lindern und ihren Patienten neue Bewegungsräume aus einer familiären und gesellschaftlichen Einschränkung eröffnen.

veau Schmerzlicher (SL) und Nichtschmerzlicher (NSL) in Deutschland, $\mathrm{n}=602$, schmerzliche Männer erwarten $41 \%$, schmerzliche Frauen 37\% mehr Schmerzen als Nichtschmerzliche
Schmerzlichen liegt in der deutschen Bevölkerung bei $50 \%(n=582)$. Die Schmerzlichen erwarten aber nicht nur bei diesen 4 genannten Krankheiten einen stärkeren Schmerz, sondern liegen in ihren Einschätzungen bei allen Krankheiten über dem Schmerzniveau der Nichtschmerzlichen ( $\bullet$ Abb. 1). In unserer Untersuchung beurteilten Nichtschmerzliche die Schmerzen von 24 vorgegebenen Krankheiten im Mittel mit 3,9 (Männer) bis 4,1 (Frauen) auf einer numerischen Analogskala (NAS) ein; Schmerzliche dagegen mit 5,5 (Männer) bzw. 5,6 (Frauen). So liegen generell schmerzliche Männer $41 \%$ und schmerzliche Frauen $37 \%$ über dem Niveau von Nichtschmerzlichen. Unserer These nach reflektieren Schmerzliche die Bedeutung einer Krankheit für ihre Vitalität und Lebensfreude sowie ihre Arbeitskraft und Bewegungsfähigkeit stärker und erleben deshalb die emotionalen Faktoren des Schmerzes stärker. Schmerz ist nicht nur ein körperliches Phänomen, er ist das emotionale Erleben, das wir die Schmerzlichkeit des Schmerzerlebens [5] nennen und das bei chronischen Schmerzen das Leiden ausmacht. Krankheit oder Schmerz werden bei schmerzlichen Patienten umso schmerzlicher erlebt, je mehr der gewohnte vitale körperliche, familiäre, gesellschaftliche und seelische Lebensvollzug als eingeschränkt empfunden wird.

Eine hohe Schmerzerwartung geht mit einer erhöhten Schmerzlichkeitseinschätzung einher. Ein Rangordnungsvergleich zeigt weiter, dass eine erhöhte Schmerzerwartung fast ausschließlich auf emotionale Faktoren des Schmerzes zurückgeht, die zusätzlich zur gesamten Schmerzeinschätzung körperlicher Schmerzen hinzuaddiert wird.

\section{Schmerzlichkeit und Kulturen}

Innerhalb der Kulturen gibt es große Unterschiede zwischen den prozentualen Anteilen der nichtschmerzlichen und der schmerzlichen Bevölkerung, wobei die untersuchten Kubaner und Griechen die emotionalen Faktoren stärker erlebten als Deutsche (• Tab. 1). Die Interviews mit den Informanten verdeutlichten, dass Menschen mit mangelnder sozialer Absicherung (fehlende Sozialversicherungen: Renten-, Kranken-, Arbeitslosen-, Berufsunfähigkeits-, Pflege-, Unfall, Risiko- und Lebensversicherungen) die Bedeutung von zu erwartenden Krankheiten anders einschätzten als diejenigen mit einer hohen sozialen Absicherung. Jede Krankheit, und sei sie nur vorgestellt, wurde vermehrt daraufhin reflektiert und beurteilt, inwieweit sie das Leben, die Arbeitsfähigkeit, das Lebensgefühl, die Vitalität und die Bewegungsfähigkeit zur Bewältigung des alltäglichen Lebens beeinträchtigen würde. Ob und inwieweit Sozialversicherungen den Menschen tatsächlich die Schmerzlichkeit des Schmerzerlebens nehmen können, ist bisher noch nicht bedacht und untersucht worden.

Der westlich ausgebildete Arzt ist hervorragend in der Behandlung der körperlichen Aspekte des Schmerzes geschult. Die Heiler in traditionell lebenden Kulturen entwickelten spezielle Methoden zur Beeinflussung des emotionalen Schmerzaspekts: Ablenkung, Umdeutung

\section{Schmerzbewältigung und Kulturen}

In allen Kulturen haben Menschen Methoden entwickelt, Krankheiten und Schmerzen zu behandeln. Dieses uralte Wissen, das oft in kulturelle Heilriten eingebettet ist, nennt die ethnomedizinische Forschung die kulturellen Krankheitsund Schmerzbewältigungsstrategien. Je nach Kultur werden bei Schmerzen unterschiedliche Verhaltensweisen beobachtet, die wesentlich von der Kontrollüberzeugung in den einzelnen Ethnien geprägt sind. Man unterscheidet zwischen:

- individualorientierten Gesellschaften (Deutsche, Briten, Iren, Nordeuropäer und Nordamerikaner) und

- familienorientierten Gesellschaften (Italiener, Türken, Mittelmeervölker, Asiaten).

Familienorientierte Gesellschaften sind überzeugt, Krankheit und Schmerz nur mit Hilfe der Familie bewältigen zu können. Patienten werden beim Arztbesuch notwendigerweise von vielen Angehörigen begleitet. Sie haben eine hohe externale Kontrollüberzeugung, während $\mathrm{Pa}$ tienten aus individualorientierten Gesellschaften überzeugt sind, sich selbst helfen zu können, indem sie der Vernunft folgend fachärztlichen Rat einholen.

Dies hat entscheidende Bedeutung für die Compliance. Bei Patienten aus familienorientierten Gesellschaften sollte der Arzt rechtzeitig darüber nachdenken, ob die Familie des Patienten in die Behandlungsmaßnahme mit einbezogen werden muss. Er kann nicht davon ausgehen, dass sein Patient eine teure Therapie oder eine Behandlung, bei der der Ehepartner mit einbezogen werden muss, allein in seiner Familie durchsetzen kann, insbesondere wenn er (oder sie) keine Vormachtstellung in der Familie hat. Hier wird der Arzt mit der Familie oder besser mit einem be- 
sonders einflussreichen Familienmitglied eigene Gespräche führen müssen.

\section{Individuelle Dimension von Krankheit und Schmerz}

Viele in fremden Traditionen erzogene Patienten erleben ihre Krankheit als ein individuelles Ereignis. Sie verstehen deshalb die Diagnose ihres Arztes nicht: Sie verstehen nicht, dass sie nun eine Krankheit haben sollen, die mit ein und demselben Begriff bezeichnet wird, wie die Krankheit anderer Menschen und dass sie z. B. nun eine Krankheit haben sollen, die auch ihr Nachbar hat. Es ist doch ihre ganz individuelle Krankheit, die sie an einem bestimmten Ort, zu einer ganz bestimmten Zeit plötzlich und unerwartet befallen hat. Der Arzt sollte deswegen bei seinen ausländischen Patienten individualisieren und nicht generalisieren.

In traditionellen Gesellschaften wird Krankheit als ein individuelles Ereignis erlebt, das den Kranken an einem bestimmten Ort zu einer ganz bestimmten Zeit getroffen hat. Der Heiler lässt sich vom Kranken ausführlich über die letzten gesunden und ersten Krankheitstage berichten, damit sie den Zeitpunkt und den Ort herausfinden, wann und wo ihn die Krankheit befallen hat (- Abb. 2). Daraufhin zieht der Heiler aus, um die verlorene Seele des Kranken wieder einzufangen oder die verletzten Ahnen und Geister durch Gebete um Verzeihung zu bitten [8]( • Abb. 3).

\section{Schmerzbewältigungsstrategien in verschiedenen Kulturen}

Im Folgenden werden verschiedene Schmerzbewältigungsstrategien vorgestellt, die inhaltlich bezeichnet sind. Stellvertretend werden ihnen einzelne ethnische oder religiöse Gruppen zugeordnet, in denen die Bewältigungsstrategie häufiger angetroffen wird. In allen Kulturen finden sich anteilmäßig verteilt alle der genannten Strategien.

Die bekanntesten 5 Schmerzbewältigungsstrategien sind:

- die fatalistische (Filipinos),

- die religiöse (gläubige Christen, Juden, Buddhisten),

- die willentliche (Iren, Indianer),

Trauma Berufskrankh 2007 · 9[Suppl 3]: S323-S328 DOI 10.1007/s10039-007-1231-6

(c) Springer Medizin Verlag 2007

N. Kohnen

Schmerzliche und nichtschmerzliche Patienten. Transkulturelle Aspekte des Schmerzerlebens

\section{Zusammenfassung}

Untersuchungen in verschiedenen Kulturen zur Schmerzwahrnehmung und zum Schmerzerleben zeigten Unterschiede v. a. im Erleben des emotionalen Schmerzaspekts, in der Schmerzlichkeit des Schmerzerlebens. Schmerzen werden unterschiedlich erlebt: je nachdem, ob ein somatisch oder ein emotional betonter Schmerzbegriff und eine familien- oder individualorientierte Kontrollüberzeugung vorliegen und je nachdem wie weit die individuell verschiedenen, erlernten und kulturell geprägten Schmerzbewältigungen durchgeführt werden können. Deutsche Ärzte haben aus ihrer soziokulturellen Sicht eine Vorstellung, welches Schmerzerleben,

\section{Presence and absence of emotional distress in pain patients. Transcultural aspects of how pain is experienced}

\section{Abstract}

Research into how pain is perceived and experienced in different cultures has shown that there are differences especially in the way the emotional aspects of pain are experienced, i.e. in the kind and degree of emotional distress it causes. Pain is experienced differently within different cultures, depending on whether the concept of pain is somatically or emotionally oriented and the locus of control is family or individual oriented, and also on the degree to which the individually different, learned and culturally determined strategies for coping with pain can be applied. Because of their sociocultural background, Ger- welche Schmerzäußerungen und welche Schmerzbewältigungen angebracht und welche unangemessen sind. Diese bezieht sich in der Regel aber nur auf ihre Erfahrungen in einer einzigen Ethnie, nämlich in der eigenen Bevölkerung. Diese einseitige ethnozentristische Sicht kann zu Verständigungsschwierigkeit und Fehlurteilen bei der Schmerzbehandlung ausländischer Patienten führen.

\section{Schlüsselwörter}

Schmerzwahrnehmung · Schmerzerleben . Schmerzbewältigung · Ethnische Aspekte . Ausländische Patienten man doctors have a particular idea of what ways of experiencing pain, what statements about pain and what coping strategies are and are not appropriate. As a rule, however, this draws exclusively on their own experience in a particular ethnic group, i.e. their own population. This one-sided ethnocentric view can lead to difficulties in communication and to misjudgements in the treatment of foreign patients.

\section{Keywords}

Pain perception - Experience of pain - Coping with pain · Ethnic aspects · Foreign patients 


\begin{tabular}{|llll}
\hline \multicolumn{1}{|l}{ Tab. 1} & Nichtschmerzliche (NSL) und Schmerzliche (SL) in verschiedenen Kulturen \\
\hline NSL (NAS 0-3) [\%] & SL (NAS 3-10) [\%] & $\begin{array}{l}\text { Anzahl (insgesamt 974 } \\
\text { Personen) }\end{array}$ \\
\hline Österreicher & 62 & 38 & 39 \\
\hline Spanier & 62 & 38 & 65 \\
\hline Rumänen & 55 & 45 & 55 \\
\hline Filipinos & 49 & 51 & 134 \\
\hline Deutsche & 47 & 53 & 423 \\
\hline Australier & 46 & 54 & 54 \\
\hline Franzosen & 27 & 73 & 44 \\
\hline Kubaner & 19 & 81 & 130 \\
\hline Griechen & 7 & 93 & 30 \\
\hline
\end{tabular}

- die familiäre (Italiener, Türken, Mittelmeervölker),

- die rationale (Nordamerikaner: Protestanten britischer Abstammung, Nordeuropäer).

Ausländische Patienten äußern nicht nur Schmerzen auf dem Hintergrund dieser erlernten Schmerzbewältigungsstrategien, sie gebrauchen den Begriff "Schmerz“ bei mangelnder Sprachkenntnis auch als Synonym für Krankheit. Schmerzbewältigungsstrategien sind gekennzeichnet durch (Kohnen [7], S 70-75):

- eine strategische Leitüberzeugung,

- die Kontrollüberzeugung,

- eine strategische Verhaltensweise,

- das subjektive Verhalten zum Schmerz und

- eine strategische Handlungsanweisung.

\section{Fatalistische Schmerzbewältigung (Filipinos)}

Natürlich ist auch bei traditionell lebenden Filipinos eine symptomatische Behandlung von Schmerzen mit Schmerzmittel bekannt, allerdings ist ihre Kontrollüberzeugung gering und sie sind überzeugt, dass nur Gott ihnen wirklich helfen und Krankheit oder Schmerz beseitigen kann ( $\bullet$ Abb. 4). Eine solche fatalistische Einstellung hat den Vorteil, dass Krankheit keinen weiteren Stress erzeugt, ganz im Unterschied zu unseren individualorientierten Verhaltensweisen im Krankheitsfall, sofort den besten Fachmann, die beste Klinik und den führenden Therapeuten für die vorliegende Krankheit zu suchen. Filipinos verhalten sich duldend gegenüber den Schmerzen und beklagen ihr Schicksal. Die eigene Initiative, eine Diagnostik zur ursäch- lichen Behandlung des Schmerzes einzuleiten, ist dagegen gering.

In abgelegenen Gebieten werden noch magische Behandlungsformen beobachtet. Sie sind hervorragend geeignet, das Krankheits- und Schmerzerleben (den emotionalen Aspekt des Schmerzes, die Kontrollüberzeugung und die Erwartungen) zu beeinflussen, während sie keine körperliche Änderung herbeiführen $[4,6]$.

\section{Religiöse Schmerzbewältigung (gläubige Christen, Juden, Buddhisten)}

Was geschah mit dem armen Hiob im Alten Testament? Gott sandte ihm Schmerz und Krankheit, um ihn zu prüfen, ob er standhaft im Glauben sei. Was wollte Gott ihm damit sagen? Es war für den Gläubigen die einmalige Chance, sein Leben nach Prüfung zu ändern und einen anderen Lebensweg einzuschlagen.

Krankheit und Schmerzen können Zeichen Gottes sein, einen heilvolleren Lebensweg einzuschlagen. Man verhält sich zum Schmerz so, dass er ertragen und erduldet werden muss, damit das Zeichen und die Botschaft Gottes erkannt werden. Er wird geäußert und er ist lästig, weswegen Wehklagen durchaus erlaubt ist, aber er sollte deshalb nicht durch Medikamente beseitigt oder völlig unterdrückt werden. So wurde beobachtet, dass die Schmerztabletten bei gläubigen Juden oft vom Nachttisch des Krankenbetts verschwanden, ohne eingenommen zu werden [13]. Schmerzen erhalten einen besonderen Sinn. Auch gläubige Moslems deuten Schmerz und Krankheit als Zeichen ihres Gottes. Buddhisten glauben an die Wirksamkeit des Karmas, das als die Summe der guten und schlechten Taten angese- hen wird. Derjenige, der hier auf Erden viel Leid erduldet, wird im Jenseits dafür reichlich entlohnt werden [10]. Dies sind 2 Beispiele für ein hervorragendes „reframing“. Das bedeutet: Ein schmerzhaftes Erleben wird in ein anderen Sinnzusammenhang gestellt und dadurch als nicht mehr so schmerzlich erlebt.

\section{Sozial geleitete und willentliche Schmerzbewältigung (Iren, Indianer)}

Die strategische Leitüberzeugung der willentlichen Schmerzbewältigung ist: Ich werde den Schmerz nicht zulassen und falls er auftritt, ihn unterdrücken. Folglich werde ich mich in die Einsamkeit zurückziehen und den Schmerz ertragen. Die Kontrollüberzeugung lautet: Ich alleine werde mit meinem Willen den Schmerz bewältigen. Zunehmende Selbstkontrolle (Kompetenzvertrauen, ,self-efficacy“[1]) und das Erleben von Vorhersagbarkeit und Kontrollierbarkeit sind als effiziente Schmerzregulationsstrategien bekannt. Unter den Iren ist es „unfein, Schmerzen zu äußern“. Man ist überzeugt, dass Gott den Sünder mit Krankheit und Schmerz bestraft. Man zieht sich zurück, damit zur körperlichen Pein nicht auch noch die gesellschaftliche Verachtung hinzukommt. Diese Art der Schmerzbewältigungsstrategie zeugt von einer starken Selbstbeherrschung und dem Einfluss der Werte und Normen, die in einer Gesellschaft vermittelt und erlernt werden können.

Und warum empfinden Indianer keinen Schmerz? Bestand zwischen 2 Indianerstämmen Krieg und ein Mitglied des gegnerischen Stammes wurde gefangen genommen, quälte man ihn am Marterpfahl. Dies war eine einfache und wirksame Messmethode, um herauszufinden, wie tapfer die Gegner waren und wie viel Entbehrungen sie aushalten würden. Es war das Maß zur Einschätzung der Gefährlichkeit und der Stärke des Gegners. Deshalb lernten die indianischen Krieger früh, ihren Schmerz nicht zu äußern.

\section{Familiäre Schmerzbewältigung (Italiener, Türken)}

Die traditionelle Form der Lebensbewältigung bei Italienern und Türken ländlicher Herkunft lautet: Schwierige Lebenssituationen werden durch Unterstüt- 
zung der Familie bewältigt. Bei Krankheit und Schmerzen wenden sich traditionell lebende Italiener deshalb an die Familie. Sie sind überzeugt, dass diese bei der Lebens- und Schmerzbewältigung durch familiäre Unterstützung und soziale Zuwendung hilft. Nun kann nur demjenigen geholfen werden, der seine Hilfsbedürftigkeit laut und deutlich äußert. Das Verhältnis zu Schmerzen ist also dadurch gekennzeichnet, dass sie zugelassen werden und ihr Erleben laut gegenüber anderen geäußert wird. Die Handlungsanweisung beim Erleben von Schmerzen lautet: die Notwendigkeit oder den Wunsch nach sozialer Zuwendung anderen deutlich präsentieren. Im Mittelmeerraum, bei christlichen sowie auch moslemischen Gruppen, ist diese Schmerzbewältigungsstrategie weit verbreitet.

\section{Rationale Schmerzbewältigung (Nordamerikaner: Protestanten britischer Abstammung, Nordeuropäer)}

Die Kontrollüberzeugung lautet: Wenn ich meine Schmerzen so präzise wie möglich beobachte und dem Arzt beschreibe, kann er am sichersten die Lokalisation der Gesundheitsstörung herausfinden, die Krankheit diagnostizieren und eine ursächliche Therapie einleiten. Diese Gruppen sind überzeugt, dass Schmerzen technisch und fachlich bewältigt werden sollen. Dies setzt ein bestimmtes Verhalten gegenüber den Schmerzen voraus: Ich werde sie nüchtern ohne emotionale Beteiligung objektiv beobachten und schildern. Sofortiges Handel und Einholen einer fachlichen Beratung ist angesagt.

\section{Fazit}

Die Unterschiede zwischen den individual- und familienorientierten Krankheitsbewältigungsstrategien sowie den kulturell verschiedenen Schmerzbewältigungsstrategien sollte der praktizierende Arzt bedenken, denn das Verweigern der erlernten kulturellen Bewältigungsstrategien führt zu Hilflosigkeit, Verzweiflung, erhöhtem Stress und damit zur Verschlechterung des Befindens in der Krankheit und zu größerer Schmerzempfindlichkeit. Transkulturelle Kommunikation ereignet sich im Zuhören, Er-
Abb. 2 - Heiler bei der Anamnese, Krankheit als individuelles Ereignis: An einem bestimmten Tag, zu einer bestimmten Stunde ist dieses Individuum von dieser Krankheit ereilt worden

Abb. 3 Heiler mit Ritualtuch und Ritualspeer auf der Suche nach der verlorenen Seele

Abb. 4 Junge kranke philippinische Frau vor Hausaltar, Gott um Hilfe bittend
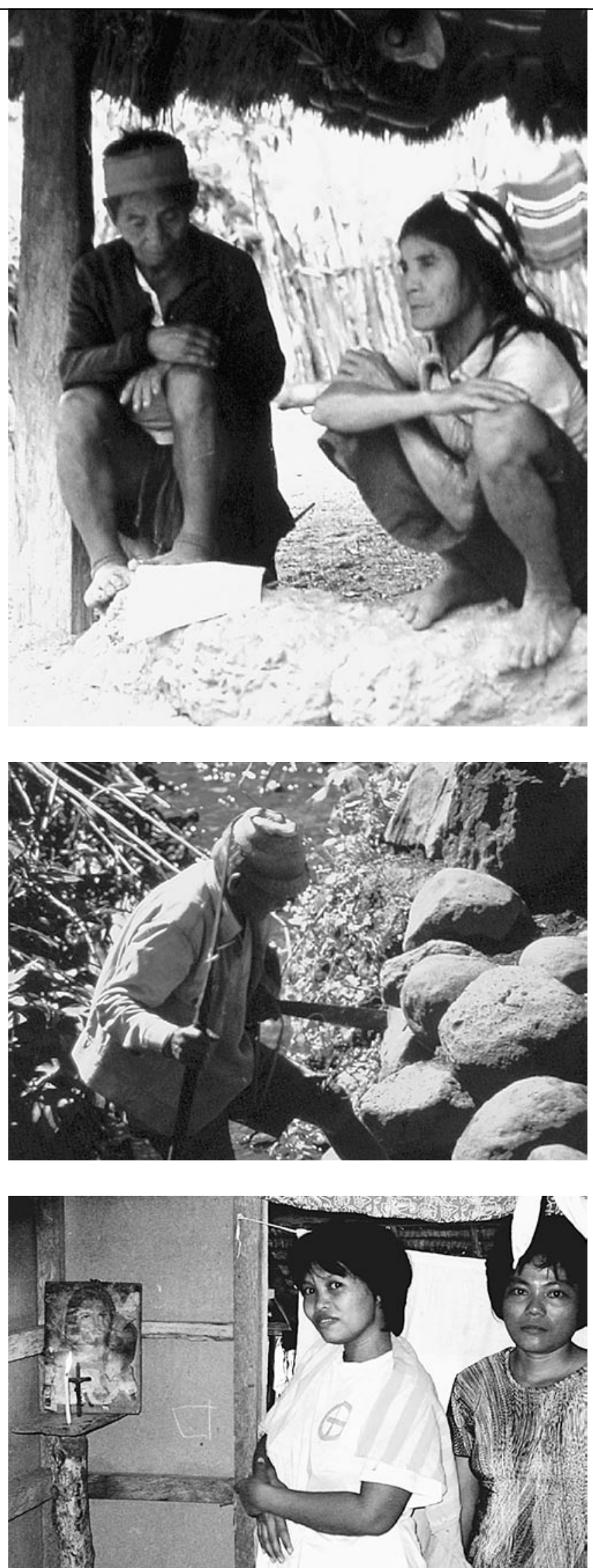
fassen und Erlernen andersartiger, wenn auch laienhafter Erklärungsweisen von Krankheit und Schmerz. Ärzte können sich in die Erlebniswelten ihrer fremdländischen Patienten eindenken, wenn sie von dem alleinigen Gültigkeitsanspruch der wissenschaftlichen Krankheitserklärungen absehen und sich auf das Denken der Patienten einlassen, um sie zu verstehen.

Jeder Patient ist ein Informant, doch nicht jeder Informant ist ein guter Informant. Ob er gut ist, liegt am untersuchenden Arzt und wie er es versteht, den Denk- und Erlebenshorizont des Informanten zu eröffnen und zu erfahren. So wird bei der Untersuchung ausländischer Patienten eine transkulturelle Kompetenz gefordert.

\section{Korrespondenzadresse}

\section{PD Dr. N. Kohnen}

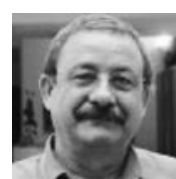

Werderstraße 31,

50672 Köln

Kohnen@uni-duesseldorf.de

Interessenkonflikt. Der korrespondierende Autor gibt an, dass kein Interessenkonflikt besteht.

\section{Literatur}

1. Bandura A (1978) Self-efficacy: toward a unifying theory of behavioral change. Adv Behav Res Ther 1: 139-161

2. Clark WC, Clark SB (1980) Pain responses in Nepalese porters. Science 209: 410-412

3. Hardy JD, Wolff HG, Goodell H (1952) Pain sensations and reactions. Williams \& Wilkens, Baltimore

4. Kohnen N (1986) Igorot. Alltag und traditionelle Wege des Heilens bei philippinischen Bergstämmen, 1. Aufl. Triltsch, Düsseldorf

5. Kohnen N (1990) Schmerzerleben der Cabuntogueños. Faktoren des Schmerzes in einer philippinischen Fischergruppe. Natur Ganzheitsmed 3: 246-251

6. Kohnen N (1992) Traditionelle Medizin auf den Philippinen. Angstbewältigung und Kognition bei Krankheit. Beiträge zur Südasienforschung. Südasieninstitut, Universität Heidelberg, Bd 154. Steiner, Stuttgart

7. Kohnen N (2003) Von der Schmerzlichkeit des Schmerzerlebens. Wie fremde Kulturen Schmerzen wahrnehmen, erleben und bewältigen. pvv-Verlag, Ratingen

8. Kohnen N (2007) Schmerzäußerungen bei fremdländischen Patienten (1). Orthodoc 1: 34-36

9. Melzack R (1978) Das Rätsel des Schmerzes. Hippokrates, Stuttgart

10. Reichwald C (2003) Ethnomedizinische Untersuchungen bei emigrierten Burmesen über die menschliche Anatomie und Krankheiten. Med. Dis sertation, Universität Düsseldorf
11. Sternbach RA, Tursky B (1965) Ethnic differences among housewives in psychophysical and skin potential responses to electric shock. Psychophysiology 1: 241-246

12. Sternbach RA, Murphy RW, Timmermans G et al. (1974) Measuring the severity of clinical pain. Adv Neurol 4: 281-295

13. Zborowski M (1952) Cultural components in responses to pain. J Soc Issues 8: 16-30 